\title{
Perforated Sigmoid Diverticulitis in the Presence of Toxic Epidermal Necrolysis
}

\author{
$\begin{array}{llll}\text { P. Heye } & \text { A. Descloux } & \text { G. Singer } & \text { R. Rosenberg } \\ & \text { a } & \text { T. Kocher }\end{array}$ \\ Departments of ${ }^{\mathrm{a}}$ Surgery and ${ }^{\mathrm{b}}$ Pathology, Kantonsspital Baden, Baden, Switzerland
}

\section{Key Words}

Diverticulitis · Sigmoid · Toxic epidermal necrolysis · Stevens-Johnson syndrome

\begin{abstract}
Even though the incidence of toxic epidermal necrolysis (TEN) is low, it is also associated with a high mortality rate. The condition predominantly affects the skin, but may also affect the gastrointestinal tract, dramatically increasing mortality. We present a case of perforated sigmoid diverticulitis in the presence of TEN. The patient was taking medication, known to be a risk factor, and presented an affected total body surface area and temporal development similar to previously reported cases of TEN. Characteristic abdominal symptoms, however, were missing. Gastrointestinal involvement in TEN appears to be a poor prognostic factor; medical staff must therefore be alert to patients with TEN who complain of abdominal discomfort. The exact pathogenesis, however, remains unclear.
\end{abstract}

\section{Introduction}

Sigmoid diverticulitis is a common disease of the lower gastrointestinal tract. Sigmoid diverticulosis has a prevalence of up to $60 \%$ in 70 -year-old individuals, of whom $10-25 \%$ may progress to diverticulitis and a further $10-25 \%$ of these may develop complications such as abscess formation, perforation or fistulas [1]. The mortality rate has been demonstrated to be $0.5-5 \%$. The most common symptoms include left lower abdominal pain lasting for a few days, fever, nausea and vomiting, and abdominal tenderness and rigidity during clinical examination. A typical sigmoidal diverticulum is a 'false diverticulum', e.g. only the mucosa and submucosa herniate through the muscular layer of the gut. Risk factors include, among others, obesity and chronic constipation. The pathophysiological process of sigmoid 
Heye et al.: Perforated Sigmoid Diverticulitis in the Presence of Toxic Epidermal Necrolysis

diverticulitis is primarily thought to be erosions caused by pressure exerted in the sigma, in combination with feces and microbial toxins which form microabscesses. These erosions develop into ulcers which may then perforate, either covered by peritoneum, or freely, into the abdominal cavity.

Toxic epidermal necrolysis (TEN) is caused by a severe inflammatory reaction, most probably of autoimmune origin. The most common triggers are antibiotics, nonsteroidal anti-inflammatory drugs and anticonvulsants. Outbreak is assumed to occur within 1-3 weeks after the first drug intake. It is characterized by cytotoxic T-lymphocytes and other cytokine pathways, causing keratinocyte apoptosis and thereby disruption of the epidermis from the dermis of the skin. However, the exact molecular and cellular pathophysiology remains unclear. It presents clinically with fever, itchy eyes, pain upon swallowing and cutaneous efflorescences such as erythematous purpuric macules on the trunk and proximal upper extremities as well as on the lips and oral, conjunctival and genital mucosae. The epidermal skin lesions progress to full-walled necrosis, materializing as epidermal detachment and resulting in blisters. A positive Nikolsky sign can be elicited, and the skin resembles wet cigarette paper when detached [2].

Stevens-Johnson syndrome affects less than $10 \%$ of the total body surface area (TBSA) and has an incidence of approximately 6 cases per million persons per year, with an average mortality rate of $1-5 \%$. TEN involves more than $30 \%$ of the TBSA and affects approximately 2 persons per year with an average mortality rate of $25-50 \%[2,3]$.

\section{Case Report}

A 91-year-old female was admitted to our Emergency Department shortly after having been found lying in the shower at home. The patient had lived by herself up to that point and presented with a medical history of arterial hypertension and spinal arthrosis. She had been taking mefenamic acid and paracetamol, but the latter had been changed to metamizole - a pyrazolone derivative - approximately 3 weeks prior to admission. Taking the patient's history was complicated by her age and dementia, and retrospectively, possibly due to a delirious state. On admission, the patient reported having suffered burns from a stove. She then took a shower where she slipped and fell, and was burnt additionally by the hot water. She denied any other respiratory, gastrointestinal and musculoskeletal complaints. Clinically, she presented with epidermal lysis on both thighs and proximal upper extremities, totaling approximately $7 \%$ of the TBSA, which in the context of the reported burning, were interpreted as second-degree burns (fig. 1). A brief debridement and wound dressing was performed. The skin showed no adherence to the underlying tissue and could be stripped off without effort. A slight reddish rash was noted on both breasts and the lower abdomen and was interpreted as a reaction to the bandages. In addition, a contusion on the left hip was diagnosed. The patient denied any abdominal pain. She presented with a distended abdomen, tender to the touch in the left lower quadrant, lacking rebound tenderness. Laboratory studies showed elevated C-reactive protein of $169 \mathrm{mg} / \mathrm{l}$ and leucocytes of $33,100 / \mu \mathrm{l}$. Moreover, she presented with lymphocytopenia (lymphocyte count $500 / \mu \mathrm{l}$ ), renal insufficiency (creatinine $121 \mu \mathrm{mol} / \mathrm{l}$ ) and a hypokalemia level of $2.7 \mathrm{mmol} / \mathrm{l}$. She tested positive for a nitrite-positive urinary tract infection. An abdominal CT scan was performed. Free air in the abdominal cavity and signs suggestive of perforated sigmoid diverticulitis were observed (fig. 2). The condition was managed by emergent sigmoid colectomy with colostomy (Hartman's procedure). In the course of intubation and surgery, a rapid progression of the epidermal detachment was noted on the abdomen, spreading to $30-40 \%$ of her 
TBSA. A direct Nikolsky sign could be elicited, and an attempt at an indirect sign was negative. Following surgery, the patient was treated with piperacillin/tazobactam (blood cultures remained negative). Renal function deteriorated progressively. During the following 2 days, further cutaneous vesicles developed, the epidermolysis generalized and the patient became increasingly delirious. After 3 days, she showed signs of apneic breathing, after which we decided on palliative treatment. The patient passed away 3 days after admission.

\section{Discussion}

The matter under discussion is whether the perforated sigmoid diverticulitis in our patient developed due to the epidermolysis resulting from an adverse reaction to medication, or whether it evolved independently.

A review of the literature shows very few cases of simultaneous TEN with ulcerative gastrointestinal disease, and only 1 case of TEN with perforated sigmoid diverticulitis. A study from 1991 by Chosidow et al. [4] reported 4 cases of TEN associated with severe intestinal symptoms. Comparable to our patient, the average TBSA affected was $37 \%$. These patients, however, underwent upper or lower gastrointestinal endoscopy for workup. Three out of the 4 patients showed severe mucosal erosive lesions in the small intestine; similar changes were also found in the colon of all patients. Epithelial cell necrosis could be observed predominantly in the sigmoid and rectum, resulting in detachment of the epithelial lining.

Similarly, very few other cases have been published describing superficial erosions with bleeding throughout the colon in the context of TEN [5-11]. These cases, however, were not complicated by perforation of the colon, as ours was.

To date, only 1 case of sigmoid perforation in the presence of TEN has been reported, by Carter and Mitchell [12]. The patient presented with cutaneous changes characteristic of TEN, in addition to renal insufficiency. In the course of 1 week, the patient complained of worsening abdominal pain associated with coffee-ground emesis and blood per rectum. A sigmoidoscopy demonstrated a diffuse inflammation with ulcerations along the sigmoid and rectum. An abdominal CT scan revealed a pneumoperitoneum, upon which surgical exploration was carried out. Fecal peritonitis, complete necrosis of the terminal ileum as well as multiple colonic perforations along the sigmoid and cecum were identified. An ileal resection and total colectomy were performed. Histological workup of the resected specimen demonstrated complete necrosis of the ileum and focal necrosis of the colon, with several areas of linear mucosal ulcerations showing lymphohistiocytic inflammatory infiltrate. After a long course of antibiotics and supportive treatment, the patient recovered and was discharged from the hospital.

There are very few reported cases of perforated ulcerative conditions in the gastrointestinal tract due to mucosal necrolysis. Some authors suggest a similar immunologic pathogenesis as for toxic epidermal necrolysis [11, 12]. Endoscopic biopsies have shown an increase in lymphoid cells, with no abnormal number of neutrophils or existence of crypt abscesses [13]. This constellation suggests a similar pathogenesis as in TEN. However, transmural perforation may be indicative of an - possibly simultaneous - infectious process such as sigmoid diverticulitis.

Our patient had no previous gastrointestinal complaints and had never undergone a colonoscopy. The resected sigmoid tissue did show preexisting diverticula, increasing the risk of diverticulitis. Histological workup demonstrated pathological changes characteristic of diverticulitis, but no lymphocytic infiltrate characteristic of TEN [14]. However, our 
patient had lymphocytopenia, possibly indicating migration of lymphocytes to dermal tissues. She presented with $30-40 \%$ affected TBSA, matching the reported mean. Also, her medication had been changed from paracetamol to metamizole approximately 3 weeks prior to developing TEN, corresponding to the time average reported so far.

In all cases reported to date, patients presented with abdominal pain, bloody diarrhea or blood per rectum, usually simultaneous to the development of skin lesions. Diagnosis was supported by colonoscopy showing mucosal erosions, predominantly in the sigmoid and rectum. Our patient did not present with the abovementioned symptoms, and colonoscopy could not be performed due to the upcoming surgery.

Only Carter and Mitchel [12] have reported free sigmoid perforation in the presence of TEN from which the patient recovered successfully. Patients discussed in other cases, as well as ours, died, proving the severity of the condition. The condition's outcome can be estimated using the SCORTEN score. Our patient acquired a score of 4 points (age $>40$ years, TBSA affected $>10 \%$, serum urea nitrogen $>28 \mathrm{mg} / \mathrm{dl}$, bicarbonate $<20 \mathrm{mmol} / \mathrm{l}$ ), which is associated with a mortality of $60 \%$ [15]. This may be indicative that an early and aggressive surgical approach is crucial in order to successfully avoid abdominal sepsis and increase survival rates.

Our patient was taking medication that presented a risk for the development of TEN. She presented with an affected TBSA and temporal development similar to previously reported cases of TEN with intestinal involvement. Characteristic abdominal symptoms, however, were missing.

In conclusion, TEN has a low incidence rate, but continues to result in serious, potentially fatal outcomes. Although the condition predominantly affects the skin, other areas such as the oral, esophageal as well as colonic (especially the sigmoid) mucosae may be affected. It has been shown that gastrointestinal involvement may increase mortality dramatically by rapidly progressing to transmural ulcerations causing fecal peritonitis. Medical staff must therefore be alert to all patients with TEN who complain of abdominal discomfort.

\section{Disclosure Statement}

There was no sponsorship and no conflicts of interest.

\section{References}

1 von Rahden BH, Germer CT: Colonic diverticulosis and its complications: pathogenesis, classification and clinical implications (in German). Zentralbl Chir 2013;138(suppl 2):e81-e85.

2 French LE: Toxic epidermal necrolysis and Stevens-Johnson syndrome: our current understanding. Allergol Int 2006;55:9-16.

3 Lissia M, Mulas P, Bulla A, Rubino C: Toxic epidermal necrolysis (Lyell's disease). Burns 2010;36:152-163.

$\checkmark 4$ Chosidow O, Delchier JC, Chaumette MT, Wechsler J, Wolkenstein P, Bourgault I, Roujeau JC, Revuz J: Intestinal involvement in drug induced toxic epidermal necrolysis. Lancet 1991;337:928.

5 Kedward AL, McKenna K: A fatal case of toxic epidermal necrolysis with extensive intestinal involvement. Clin Exp Dermatol 2009;34:e484.

6 Roupe G, Ahlmén M, Fagerberg B, Suurküla M: Toxic epidermal necrolysis with extensive mucosal erosions of the gastrointestinal and respiratory tracts. Int Arch Allergy Appl Immunol 1986;80:145-151.

-7 Otomi M, Yano M, Aoki H, Takahashi K, Omoya T, Suzuki Y, Nakamoto J, Kataoka K, Yagi Y, Yamamoto Y: A case of toxic epidermal necrolysis with severe intestinal manifestation. Nihon Shokakibyo Gakkai Zasshi 2008;105:1353-1361.

8 Sugimoto Y, Mizutani H, Sato T, Kawamura N, Ohkouchi K, Shimizu M: Toxic epidermal necrolysis with severe gastrointestinal mucosal cell death. A patient who excreted long tubes of dead intestinal epithelium. J Dermatol 1998;25:533-538. 


\begin{tabular}{l|l}
\hline Case Rep Dermatol 2014;6:49-53 \\
\hline DOI: 10.1159/000360129 & $\begin{array}{l}\text { C 2014 S. Karger AG, Basel } \\
\text { www.karger.com/cde }\end{array}$ \\
\hline
\end{tabular}

Heye et al.: Perforated Sigmoid Diverticulitis in the Presence of Toxic Epidermal Necrolysis

9 Jha AK, Goenka MK: Colonic involvement in Stevens-Johnson syndrome: a rare entity. Dig Endosc 2012;24:382.

10 Powell N, Munro JM, Rowbotham D: Colonic involvement in Stevens-Johnson syndrome. Postgrad Med J 2006;82:e10.

11 Sakai N, Yoshizawa Y, Amano A, Higashi N, Aoki M, Seo T, Suzuki K, Tanaka S, Tsukui T, Sakamoto C, Arai M, Yamamoto Y, Kawana S: Toxic epidermal necrolysis complicated by multiple intestinal ulcers. Int J Dermatol 2008;47:180-182.

$\$ 12$ Carter FM, Mitchell CK: Toxic epidermal necrolysis - an unusual cause of colonic perforation. Report of a case. Dis Colon Rectum 1993;36:773-777.

13 Zweiban B, Cohen H, Chandrasoma P: Gastrointestinal involvement complicating Stevens-Johnson syndrome. Gastroenterology 1986;91:469-474.

14 Quinn AM, Brown K, Bonish BK, Curry J, Gordon KB, Sinacore J, Gamelli R, Nickoloff BJ: Uncovering histologic criteria with prognostic significance in toxic epidermal necrolysis. Arch Dermatol 2005;141:683-687.

15 Bastuji-Garin S, Fouchard N, Bertocchi M, Roujeau JC, Revuz J, Wolkenstein P: SCORTEN: a severity-of-illness score for toxic epidermal necrolysis. J Invest Dermatol 2000;115:149-153.

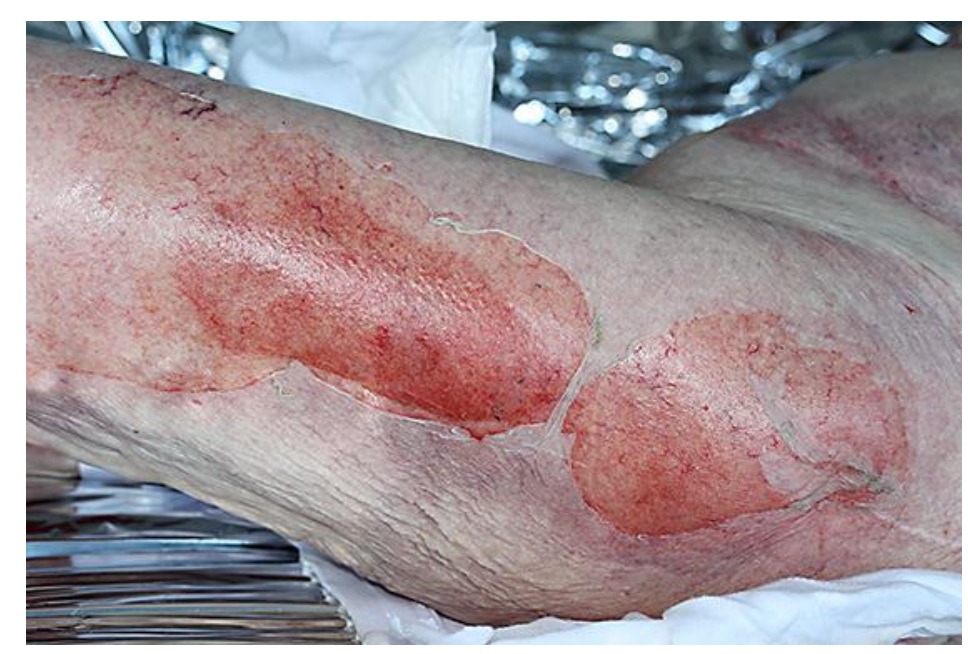

Fig. 1. Photo showing 'wet cigarette paper' skin condition on the lower extremities.

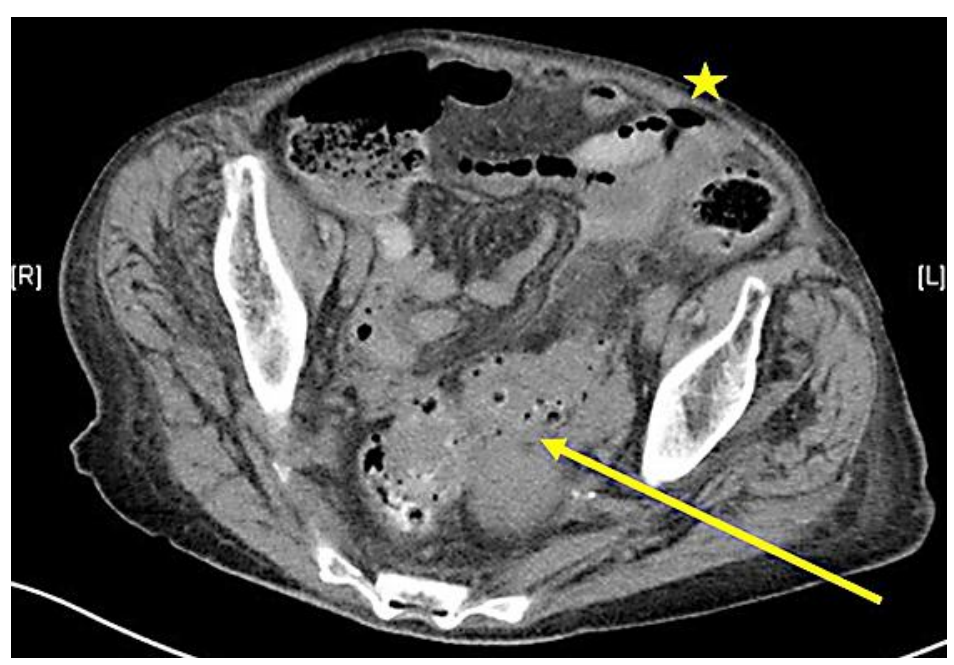

Fig. 2. Abdominal CT scan showing sigmoid diverticulitis (arrow) and free air (star). 